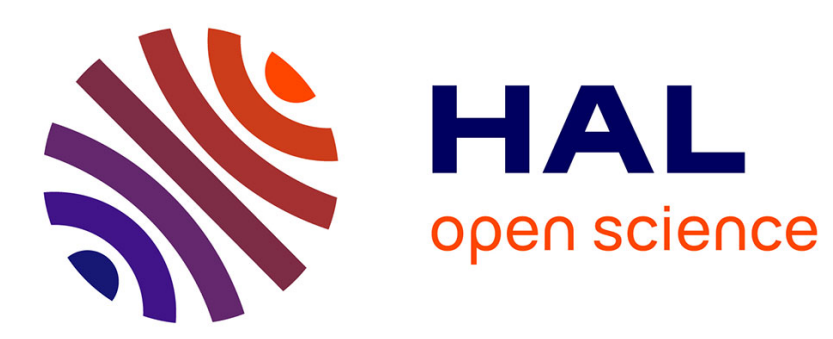

\title{
On the stability of the production of bubbles in yield-stress fluid using flow-focusing and T-junction devices
}

Benoit Laborie, Florence Rouyer, Dan Angelescu, Elise Lorenceau

\section{- To cite this version:}

Benoit Laborie, Florence Rouyer, Dan Angelescu, Elise Lorenceau. On the stability of the production of bubbles in yield-stress fluid using flow-focusing and T-junction devices. Physics of Fluids, 2016, 28 (6), pp.063103. 10.1063/1.4953678 . hal-01345195

\section{HAL Id: hal-01345195 \\ https://hal-enpc.archives-ouvertes.fr/hal-01345195}

Submitted on 13 Jul 2016

HAL is a multi-disciplinary open access archive for the deposit and dissemination of scientific research documents, whether they are published or not. The documents may come from teaching and research institutions in France or abroad, or from public or private research centers.
L'archive ouverte pluridisciplinaire HAL, est destinée au dépôt et à la diffusion de documents scientifiques de niveau recherche, publiés ou non, émanant des établissements d'enseignement et de recherche français ou étrangers, des laboratoires publics ou privés. 


\title{
On the stability of the production of bubbles in Yield-stress Fluid using Flow-focusing and $T$-junction devices
}

\author{
B. Laborie,,$^{1,2}$ F. Rouyer, ${ }^{3}$ D.A. Angelescu, ${ }^{2,4}$ and E. Lorenceau ${ }^{1}$ \\ 1) Université Paris-Est, Laboratoire Navier, UMR 8205 CNRS, ENPC ParisTech, IFSTTAR, 2 allée Kepler, \\ 77 420 Champs-Sur-Marne \\ 2) Université Paris-Est, ESIEE Paris/ESYCOM, 2 Bd. Blaise Pascal, Noisy le Grand, 93162, \\ France. \\ 3) Université Paris-Est, Laboratoire Navier, UMR 8205 CNRS, ENPC ParisTech, IFSTTAR, \\ 77454 Champs-Sur-Marne \\ ${ }^{4)}$ Fluidion SAS, 231 Rue St. Honoré, 75001, France.
}

(Dated: 13 July 2016)

We investigate experimentally the stability of bubble production in yield-stress fluids (YSF) and highly viscous silicone oil, using flow-focusing and T-junction devices. When the exit channel is initially pre-filled with the fluid and the gas is pressure-driven, the production is highly unstable, despite a regular frequency of bubble production in the junction. As observed for pressure-driven bubble trains in Newtonian fluids, we report that two mechanisms can explain these observations : $(i)$ drastic reduction of the hydrodynamic pressure drop along the channel during the transient bubble production, which induces a rapid increase of the gas flow rate and $(i i)$ thin film deposition resulting in a cascade of plug break-up and bubbles coalescence. While the drastic reduction of the pressure drop is inevitable in such two-phase flows, we show that modifying the surfaces of the channel can help stabilizing the system when the continuous phase is a YSF. To do so, we measure the thickness of the film deposited on the channel wall for rough and smooth channels. Our results are rationalized by introducing the inverse of the Bingham number $B i^{-1}$ comparing the viscous stress to the yield stress. For $B i^{-1} \geq 1$, a fast fluidization process associated to efficient deposition of YSF on the channel wall leads to a rapid destabilization of the bubble production. However, for $B i^{-1}<1$, the deposition driven by capillarity can be hindered by the wall-slip induced by the existence of the yield stress: the thickness of the deposited film is very thin and corresponds to the equivalent roughness of the channels. It is typically $40 \mu \mathrm{m}$ thick for rough surfaces and below the limit of resolution of our set-up for smooth surfaces. In this regime of $B i^{-1}$ and for smooth surfaces, the length of the plugs barely vanishes, thus the start-up flow is less prone to destabilization. These results therefore potentially open routes to steady production of aerated YSF on smooth channels in the regime of small $B i^{-1}$.

PACS numbers: 47.55.D- Drops and bubbles, 47.57.Bc Foams and emulsions, 47.55.N- Interfacial flows, 47.50.-d Non-Newtonian fluid flows, 47.15.Rq Laminar flows in cavities, channels, ducts, and conduits Keywords: Bubbles, Microfluidics, Yield-stress fluids

\section{INTRODUCTION}

Microfluidics is a widely-used technology for producing and transporting droplets and bubbles of well-controlled size $^{5,11,30}$, or for the production of complex materials, such as monodisperse foams ${ }^{23,48}$, complex emulsions ${ }^{44,45}$, or dispersions of non-Newtonian fluids ${ }^{15,29,54}$. Important progress has also been made in understanding microchannel flows ${ }^{12,17,19,28}$. These aspects are critical for producing controlled dispersions, which require simultaneous control over both the bubble/droplet break-up mechanisms, and the subsequent two-phase channel flow.

While several regimes of bubble formation have been reported ${ }^{11}$, there are only few simple flow control methods. The easiest way to achieve control over the flow is to apply either a constant volumetric flow rate or a constant pressure to each phase ${ }^{53}$. A convenient set-up for creating gas dispersions in a liquid consists of imposing the gas pressure and the liquid flow-rate. This allows to obtain well-controlled flows by fine-tuning the inlet parameters, which determine the final bubble size, thus leading to the production of uniform dispersions ${ }^{11}$. While the initial tuning of the parameters during the production start-up phase is rarely detailed in literature, this phase is critical for establishing a steady-state production regime, and is known to be strongly affected by hydrodynamic feedback that can lead to non-uniform bubble formation $20,21,23,48,50$. For Newtonian fluids, such feedback is critical during the start-up phase and can induce transient oscillation of the frequency of bubble production in flow-focusing devices ${ }^{50}$. Even when the gas pressure is barely modified, various flow patterns of bubbles or transient disordered regimes may appear, which can take several minutes to re-stabilize ${ }^{21,23,48}$. These different results highlight the sensitivity of the start-up flow in microfluidic devices to any change of operating conditions due to hydrodynamic feedback. Understanding and controlling the start-up phase is therefore crucial for any industrial production of controlled materials, especially when scaling up production using parallelized systems that are already prone to instability due to the existing feedback between their different branches ${ }^{27}$. 
At small scales, these hydrodynamic feedbacks are strongly linked to the interfacial dynamics and have been extensively modeled and studied for Newtonian fluids ${ }^{6,9,10,48}$. Much less is known if the continuous phase is a yield-stress fluid (YSF), despite obvious industrial relevance: many processes focus on adding gas bubbles to such complex materials to enhance their properties. Examples include building materials for sustainable construction (such as concrete, plaster), food products (such as whipped cream, ice cream), or cosmetic creams $^{41,51}$. Foaming these materials has various advantages: it makes building materials lighter and more insulating (thermally or acoustically) and allows to control the texture and shape of food or cosmetic products.

YSF often exhibit a microstructure consisting, e.g., of polymer chains, micro beads or fibers, microbubbles or droplets, which result in solid elastic response below, and liquid flow response above, a certain critical stress value $\tau_{y}$, called the yield stress ${ }^{7,13,43}$. Consequently, YSF flowing in circular channels may exhibit simultaneous solid and liquid behavior, under the form of a solid-like plug at the center of the channel and a sheared liquid annulus close to the wall. In addition, YSF flow in circular channels with smooth surfaces are often prone to wall slip, which can be avoided by applying a chemical treatment ${ }^{4,7}$. In this regime, the velocity profile is a full plug and the material has a solid-like consistency on almost the entire channel cross-section, which is very different from the Poiseuille-like velocity profile exhibited by Newtonian fluids. How the presence of this solid-like region, which is all the more important if the system exhibits wall slip, affects the interfacial dynamics and the start-up phase of bubble production in microfluidics channels is currently an open question.

We focus the present report on the start-up of bubble production in micro- and millifluidic devices when the continuous phase is a YSF. When using flow-focusing or $T$-junction devices, we observe the break-down of bubble production during the transient regime. We study the two processes responsible for this breakdown, namely $(i)$ important reduction of the hydrodynamic pressure drop along the channel and (ii) deposition of a thin film leading to bubble coalescence, by performing experiments on single YSF plugs as well as on bubble trains including multiple YSF plugs. This allows us to retrieve a deposition law and to probe the effect of wall slip and surface roughness on the stability of the flow during the start-up phase of bubble production. We also identify routes for reducing the instabilities, which may lead to devices capable to produce stable dispersions of bubbles in YSF on a large scale.

\section{MATERIALS AND DEVICES}

$T$-junction and flow-focusing devices have been used to study the start-up phase of bubbles production in YSF, while glass capillaries were used to probe the deposition on YSF on smooth surfaces.

\section{Devices.}

Our three dimensional $T$-junction and flow-focusing devices are made of transparent resin and manufactured (FineLine Prototyping Inc.) using stereolithography (STL). This technique relies on the local crosslinking of a photopolymer resin under the controlled displacement of a laser. The first built layer adheres to a support platform which is moved down of one step $(50 \mu \mathrm{m}$ for our devices) and covered again by the liquid polymer, the process being subsequently repeated until the whole part is built layer by layer. However, this layer-by-layer STL manufacturing process implies that the cross section of our channels is not a perfect circle but rather a discretized circular shape, resulting in controlled circumferential roughness (hereforth referred to simply as roughness). See supplementary material at [URL to be inserted by AIP] for the calculation of the surface of the channel taking into account the roughness ${ }^{1}$. Our devices have channels of radii $R=0.5,1,2 \mathrm{~mm}$, and an outlet channel length $(L)$ of 5 or $10 \mathrm{~cm}$. In the following we detail the results obtained for T-junctions (as shown in 2) but the same phenomenology was observed with flowfocusing devices. We have presented the details of bubble formation in YSF in these geometries elsewhere ${ }^{32}$.

To study the deposition of YSF in smooth channels, we use glass capillaries of length $10 \mathrm{~cm}$, and radius $(R) 235$, $513,620,702 \mu \mathrm{m}$. These capillaries possess no detectable roughness.

For all experiments, we impose the gas pressure using a Fluigent pressure controller (MFCZ 0-2 bar), and the YSF flow rate using a syringe pump (Harvard Apparatus). Movies of the experiments are recorded using a CCD camera (Marlin) at a maximum frame rate of $200 \mathrm{fps}$. The image resolution is $9.3 \mu \mathrm{m}(1 \mathrm{px})$, and the camera shutter opening time is between 100 and $600 \mu \mathrm{s}$.

\section{Materials.}

We use two model YSF: $(i)$ concentrated oil-in-water emulsions; (ii) carbopol gels; which are known to be good model of simple YSF (i.e. not thixotropic) ${ }^{14,39,42}$. They present different microstructure but obey the same nonlinear stress $(\tau)$ / shear-rate $(\dot{\gamma})$ relationship, given by the Herschel-Bulkley law in simple shear: $\tau=\tau_{y}+k \dot{\gamma}^{n}$, with $\tau_{y}$ between $100 \mathrm{~Pa}$ and $300 \mathrm{~Pa}$ for the emulsion and $75 \mathrm{~Pa}$ for the Carbopol gel, $k$ the consistency index ranging between $35 \mathrm{~Pa} . \mathrm{s}^{n}$ and $124 \mathrm{~Pa} . \mathrm{s}^{n}$, and $n$ the power law index equal to 0.5 for emulsions and 0.35 for Carbopol gel. These rheological parameters are determined using a Bohlin C-VOR rheometer with rough parallel plates, which will be hereforth referred to as "bulk" measurements. 
Our Carbopol (980 from Cooper) is made of polyacrylic acid (PAA) resins dispersed in water and neutralized with a base. The neutralization generates ionic repulsion between the polymer chains which adopt an expanded configuration, thus forming blobs that swell until the osmotic pressure inside the blob balances the pressure inside the solvent. The size of individual blobs is of the order of $100 \mu \mathrm{m}^{35,47}$. We disperse the carbopol in water at a concentration of $1.1 \%$. To neutralize the solution we use sodium hydroxide $(\mathrm{NaOH}, 1 \mathrm{~mol} / \mathrm{L})$. The mixing lasts 24 hours and is performed with a planetary mixer to ensure homogeneous mixing throughout the sample. The carbopol density $(\rho)$ is $980 \mathrm{~kg} / \mathrm{m}^{3}$, and its surface tension $(T)$ is approximately the surface tension of water: $66 \mathrm{mN} / \mathrm{m}^{8,31}$.

Our oil-in-water emulsions are made of a dispersion of silicone oil droplets into a water-glycerol immiscible phase. The glycerol quantity is adjusted to obtain a transparent material. When the droplet volume fraction $\phi \geq 0.64$, droplets are deformed by their neighbours and form a contact network that is able to handle stresses without flowing, thus becoming a YSF. We use $350 \mathrm{cP}$ silicone oil (90\% vol.) and water/glycerol mixture (47/53 wt/wt, $10 \%$ vol.). $3 \% \mathrm{wt} / \mathrm{wt}$ of surfactant (tetradecyltrimethylammonium bromide from Sigma-Aldrich) is added to the glycerol-water mixture to stabilize the interfaces. The mixing is performed using a Couette emulsifier (manufactured by TSR). The resulting mean droplet diameter, which lies in the range $5-10 \mu \mathrm{m}$, depends on the applied shear rate. The droplet size distribution of the emulsions, determined optically using a confocal microscope (Zeiss Axio Observer Z1 with LSM700 confocal lens), is in agreement with previous light scattering measurements on similar emulsions ${ }^{25}$. As suggested by previous studies ${ }^{18}$, we assume that the surface tension of emulsions is roughly the surface tension of their continuous phase with surfactant, $T=35 \mathrm{mN} / \mathrm{m}$.

For sake of comparison with Newtonian fluids, we also use Newtonian silicone oils (Fungilab RT100000) with a viscosity $\eta=100$ Pa.s.

\section{YSF FLOW IN CONFINED CHANNELS}

YSF flowing in microchannels exhibit unsual microflow response due to the commensurability of the fluid characteristic length scales with the channel's diameter. Hence, various features such as finite-size effects and non-local rheology, phase-separation or particle's migrations, and shear inhomogeneities leading to shear banding or wall slip have often been reported ${ }^{3,4,7,24,25}$.

\section{Non-local rheology, wall slip and particle's migration.}

Non-local rheology of emulsions similar than ours has been observed when the ratio of the channel diameter to the size of the microstructure is below 40 as shown $\mathrm{in}^{25}$, which is twice smaller than in our experiment. For carbopol gels, confinement effects have been reported in microchannels with radius below $150 \mu \mathrm{m}^{24}$. This value is far below our minimum channel radius of $500 \mu \mathrm{m}$, which suggests that the flow is not modified by confinement in our experiments. The results presented here should be valid for the two materials down to this confinement limit and therefore it is reasonable to use the constitutive law deduced from Capillary rheometer experiment.

Shear banding and wall slip, which are both consequences of shear inhomogeneity, may appear simultaneously ${ }^{38}$. To detect possible discontinuities present in the flow-field, we followed the velocity profile in the exit channel of a device prefilled with emulsion $\left(\tau_{y}=\right.$ $150 \mathrm{~Pa})$ using small $(6 \mu \mathrm{m})$ fluorescent particles tracking. We observed a flat uniform velocity profile across the channel for the whole range of flow-rate investigated, which is characteristic of a slip regime. We never observed shear-banding and thus we can conclude that wall slip is the only discernible form of shear inhomogeneity in our set-up.

The evidence of wall slip not a surprising feature: YSF possess a finite size microstructure, therefore it exists a depletion region near the smooth solid surfaces free of droplets or polymer blobs. This region, filled by the YSF continuous phase (or solvent) which usually possesses almost Newtonian properties can experience most of the applied stress during the material flow. This results in an apparent velocity of the material at the wall ${ }^{4,7}$.

We also report that the confinement of the flow does not seem to induce any phase separation nor any migration of blobs: the texture and the dynamics of spreading of centimetric Carbopol drops on a flat surface due to gravity are similar prior and after the flow. Blob migration is indeed not likely to happen in these systems due to the high concentration of Carbopol present in the material, which is required in order to achieve high value of yield stresses.

\section{Flow characterization via Capillary rheometry.}

Thus, to evaluate the impact of wall slip on the flow of the materials inside our different channels, we perform a capillary rheometry experiment. This allows to extract effective rheological parameters which are representative of the behavior of the considered material in the chosen channel.

First, the channel is filled with the YSF. Then, the material is pushed at constant pressure $(\Delta P)$ by an infinite gas bubble. We measure the velocity of the bubble meniscus $(V(t))$ at least $20 R$ downstream of the junction to avoid possible perturbations due to entrance effects, and the length $\left(L_{y}(t)\right)$ of the YSF remaining in the channel as the meniscus advances. Neglecting the thin film, the mean flow rate is thus given by: $Q=\pi R^{2} V$ and the wall shear stress by $\tau_{w}=\frac{\Delta P R}{2 L_{y}}$. Then, the wall shear rate $\left(\dot{\gamma}_{w}\right)$ 
is computed with the Mooney-Rabinowitsch formula ${ }^{34}$ :

$$
\dot{\gamma}_{w}=\frac{-1}{\pi R^{3}}\left(3 Q+\tau_{w} \frac{\mathrm{d} Q}{\mathrm{~d} \tau_{w}}\right)
$$

The results for carbopol gels flowing in channels with different radii and surfaces are presented in Figure 1 along with the corresponding fit to a Herschel-Bulkley constitutive equation $\tau=\tau_{y}+k \dot{\gamma}^{n}$, with $\tau_{y}$ the yield stress, $k$ the consistency parameter $\left(\mathrm{Pa} . \mathrm{s}^{n}\right)$, and $n$ the flow index. Since $k$ and $n$ are related to the flow of the liquefied material, they should not depend on the boundary conditions, therefore we choose to force the value of $k$ and $n$ to be as close as possible to their bulk values. Indeed as the shear rate increases, the material must yield causing the slip layer to be destructured, and the flow recover mostly its bulk rheology ${ }^{40}$. Under this constraint, the best fit values of the three parameters are summarized in Table I, along with the "bulk yield stress" of the materials.

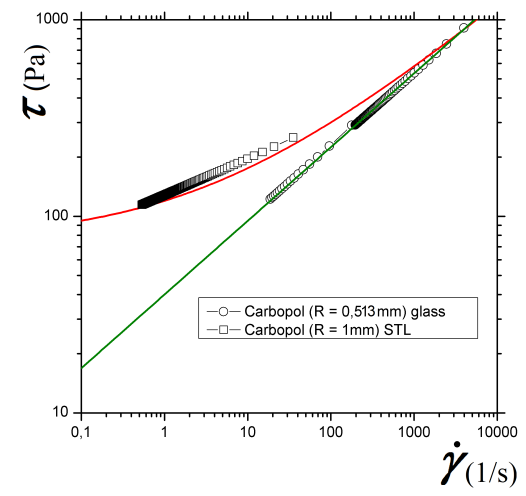

FIG. 1. Effective rheological behaviour of carbopol gel (C1) with a bulk yield stress of $75 \mathrm{~Pa}$ inside different channels. The fitting parameters of Herschel-Bulkley law are displayed in Table I

The apparent rheology of the different materials when flowing in each type of channel is different, as a consequence of wall slip ${ }^{4,34}$. We can note that: $(i)$ in all cases except for carbopol solutions in STL channels, the apparent yield stress of the material (the sliding yield stress) is lower than its bulk yield stress, a typical signature of wall $\operatorname{slip}^{49} ;($ ii $)$ when the shear rate increases, the data (and as expected, the extrapolated fits) tend assimptotically to the same rheological behaviour. A similar behaviour is observed for oil-in-water emulsions.

This underlines that the sliding yield stress measured in smooth channel is not an intrinsic property of the material but results from the fluid flow on the surface of the channel $^{40,49}$.

\section{BUBBLE PRODUCTION IN YSF}

\section{Experiments using STL rough channels.}

To investigate the start-up of bubble production, we proceed as follows: first the outlet channel of the $\mathrm{T}$ junction or flow-focusing device is completely filled with YSF at an imposed flow rate $Q$ in the range 0.01-1.5 $\mathrm{ml} / \mathrm{min}$. Then, gas pressure $(\Delta P)$ is applied to the lateral channel of the $T$-junction until it overcomes the pressure associated with the YSF flow at the imposed flow rate, which leads the onset of bubble production (Figure 2). We observe that the time to form each bubble is constant, because the devices operate in "geometrical mode" 32 : the time to produce one bubble is given at first order by $t_{b} \simeq \Omega / Q$, the time to fill the junction (of volume $\Omega$ ) at the imposed YSF flow rate $(Q)$. This regime is qualitatively similar to the quasi-static break-up mechanism observed for Newtonian fluid in 2D flow-focusing devices $^{22}$, in which the final pinch-off driven by capillarity is extremely fast compared to the slow (quasi-static) thinning of the gas thread ${ }^{52}$. However, a constant time of bubble production is not sufficient to ensure a steady production of bubbles: after a quasi-steady regime of few seconds leading to the production of nearly $402 \mathrm{~mm}$ long bubbles, the length $(\ell)$ of the successive gas bubbles quickly increases until becoming more than a centimetre long (Figure 3). Moreover, when travelling along the channel, the length of the plugs separating two adjacent bubbles, $L_{p}$, decreases. This can be visualized looking at the spatiotemporal diagram in Figure 2. To obtain this figure, we first retrieve the grey levels of each image of the experimental movie along the centerline of the outlet channel, then we stack the resulting pixel lines side by side. We observe that the coalescence of all plugs is extremely quick: it lasts $1.55 \mathrm{~s}$ compared to $\simeq 2 \mathrm{mins}$, the total duration of the bubbling regime.

Two main processes were identified as responsible for the destabilization of the bubble production: the production of successive bubbles of increasing length (Figure 3 ) and the coalescence of all bubbles due to the catastrophic thinning of the YSF plugs, which results in a co-flow of gas and YSF. We emphasize that these observations also concern the production of bubbles in Newtonian silicone oil, and are highly reminiscent of results from literature concerning the pressure-driven flow of a bubble train in a viscous fluid ${ }^{6}$ : after a transient slow flow, the viscous plugs separating the bubbles suddenly rupture and are replaced with a rapid gas flow. This has been explained ${ }^{6}$ though the combined effect of $(i)$ global interactions through hydrodynamic feedback and (ii) interactions among nearest-neighbour plugs through the wetting films. Indeed, when the train of plugs is set into motion and accelerates, the liquid plugs leave a larger amount of liquid on the channel' walls than what they may recover from the liquid film ahead of them, because the amount of liquid deposited on the wall is an increasing function of the velocity ${ }^{9}$. This leads to a 
TABLE I. Bulk yield stress and apparent rheological parameters of carbopol gels (C) and oil-in-water emulsions (E) in channels (of radius $R$ ) manufactured by stereolithography (STL) used for bubbling experiments or glass capillaries

\begin{tabular}{lllllll}
\hline Fluid & surface & $R(\mathrm{~mm})$ & $\tau_{y}(\mathrm{~Pa})-$ bulk & $\tau_{y}(\mathrm{~Pa})$ - sliding & $k\left(\mathrm{~Pa} . \mathrm{s}^{n}\right)$ & \multicolumn{2}{l}{${ }^{2}$} \\
\hline C1 & STL & 1 & $75 \pm 10 \%$ & $75 \pm 10 \%$ & $0.35 \pm 10 \%$ \\
C1 & glass & 0.513 & $75 \pm 10 \%$ & 0 & $40 \pm 15 \%$ & $0.375 \pm 10 \%$ \\
E1 & STL & 1 & $100 \pm 10 \%$ & $9 \pm 10 \%$ & $78.5 \pm 15 \%$ & $0.5 \pm 10 \%$ \\
E2 & glass & 0.513 & $150 \pm 10 \%$ & $3.1 \pm 10 \%$ & $50 \pm 15 \%$ & $0.59 \pm 10 \%$ \\
\hline
\end{tabular}

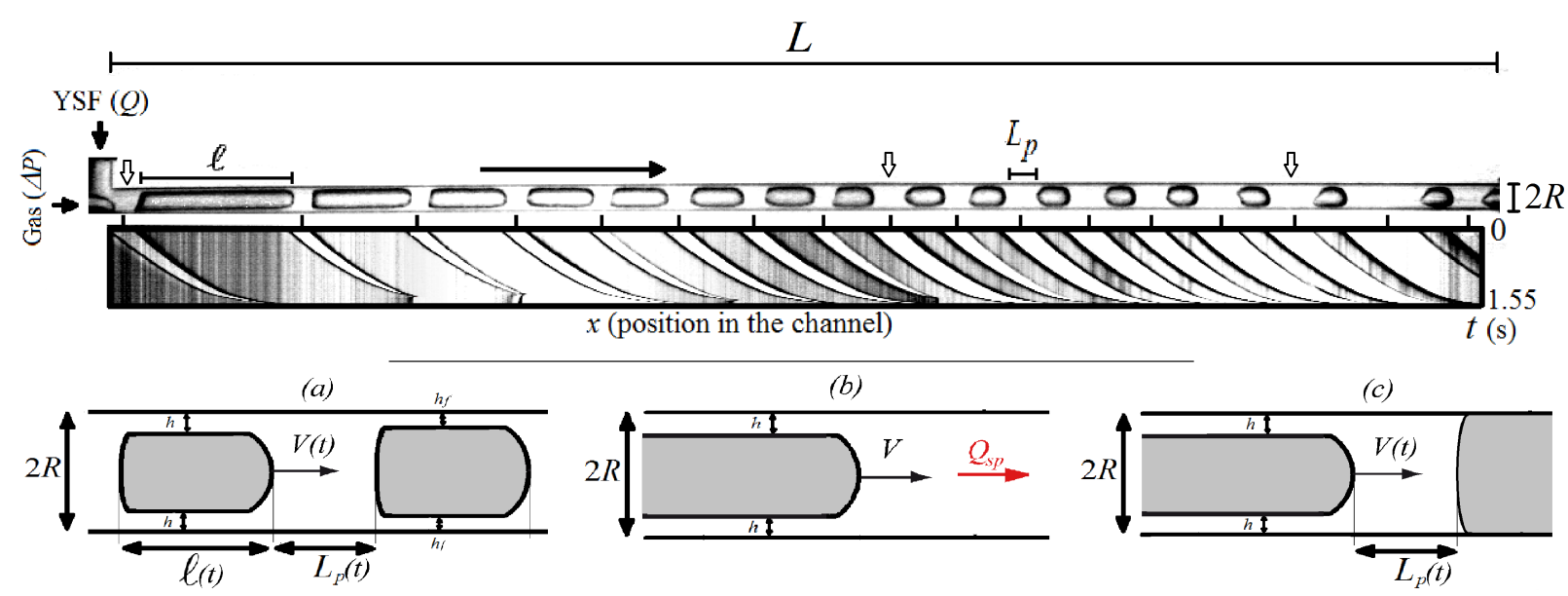

FIG. 2. Top: initial configuration and corresponding spatiotemporal diagram for plug rupture visualization and measurement of $L_{p}$ (carbopol C1 in a STL channel: $R=1 \mathrm{~mm}, L=10 \mathrm{~cm}$, with $Q=0.05 \mathrm{ml} / \mathrm{min}$ and $\Delta P=297$ mbar). The temporal evolution is visualized on the centerline of the channel. Bottom: sketch of experimental measurements: a) plug of YSF between two bubbles following each other in a multiple plugs experiment; b) meniscus displacement in steady single plug experiment at imposed flow rate; c) plug displacement between two "infinite" bubbles in unsteady single plug experiment

decrease in the plugs' length, which in turn leads to a decrease of the global viscous resistance, which leads to fast increase in the plugs' velocity under the effect of the constant gas pressure. Finally, when one plug breaks, the pressure drop decreases strongly and the flow accelerates thus leading to a cascade of plug rupture. Due to the simple rheology of Newtonian fluids, this effect has been quantitatively modeled ${ }^{6}$. In the following, we propose to adapt this scenario to understand, the start-up phase of the production of bubbles in a YSF when using T-junction or flow-focusing devices.

\section{Hydrodynamic feedback: increase in bubble size.}

We first propose to interpret the increase in bubbles' size as an hydrodynamic effect due to the modification of the hydrodynamic resistance downstream of the junction by bubbles flowing in the outlet channel. For Newtonian fluids, such approach allows to describe the size of the bubbles produced in flow-focusing devices ${ }^{21}$, and also to take into account the effect of the extra-pressure drop associated with the presence of bubbles in the outlet channel of the device ${ }^{50}$, as well as the pattern observed during foam production in microchannels ${ }^{48}$. In these cases, the pressure drop associated with the two-phase flow is due to ${ }^{5}$ : $(i)$ the viscous contribution due to the plugs sepa- rating each bubble; $(i i)$ the pressure drop due to the interfaces at the front and rear of each bubbles. For Newtonian fluids in microfluidics geometries the interfacial contribution increases the total hydrodynamic resistance $\left(r_{h}(t)\right)$ compared to a single phase flow of the same liquid. If the gas is driven at imposed pressure $(\Delta P)$, this leads to a decrease in gas flow rate $Q_{g}(t)=\Delta P / r_{h}(t)$. Finally, considering that the time to form a bubble $\left(t_{b}\right)$ is approximately constant, and since $\ell(t) \sim \frac{Q_{g}(t) t_{b}}{\pi R^{2}}$ it should imply the production of bubbles of decreasing length.

Yet, on Figure 3, we observe an increase in bubble size during the experiment, therefore implying a decrease in total hydrodynamic resistance. We propose to explain this result by considering: $(i)$ the large effective viscosity of YSF compared to Newtonian fluids, which tends to make dominant the role of the viscous pressure drop inside the plugs; (ii) the relatively large size of our channels compared to microfluidics channels that tend to decrease the interfacial pressure drop, which becomes all the more negligible compared to the viscous contribution. Therefore, if the viscous contribution is dominant, as a bubble is formed, the volume fraction of gas inside the channel increases, in turn decreasing its hydrodynamic resistance $\left(r_{h}(t)\right)$. Since the gas is driven at imposed pressure and the time to form a bubble $\left(t_{b}\right)^{32}$ is approximately constant, it implies the production of bubbles of increasing length. 


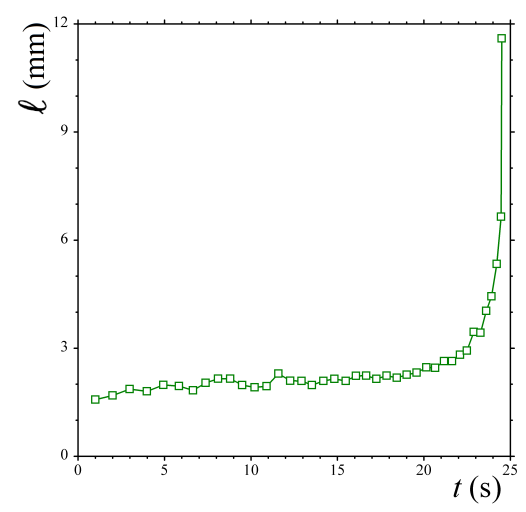

FIG. 3. Length $(\ell)$ of the successive bubbles formed in a $T$ junction with $Q=1.25 \mathrm{ml} / \mathrm{min}, \Delta P=0.62 \mathrm{bar}, R=1 \mathrm{~mm}$, and $L=10 \mathrm{~cm}$. The lengths are measured at the position $x=0$ and are reported as a function of the time $(t)$ passed since the beginning of the experiment

While these assumptions account well for the observed unstable behavior, they do not take into account the fluid deposition, and subsequent bubble coalescences that lead to the break down of the bubbling regime.

\section{Fluid deposition: plug rupture.}

To quantify the effect of the fluid deposition over the destabilization, we follow the evolution of the lengths of three plugs $\left(L_{p}\right)$ as they flow in the channel along with the flow mean velocity $(V)$. These are reported as a function of time from the beginning of the experiment on Figure 4.

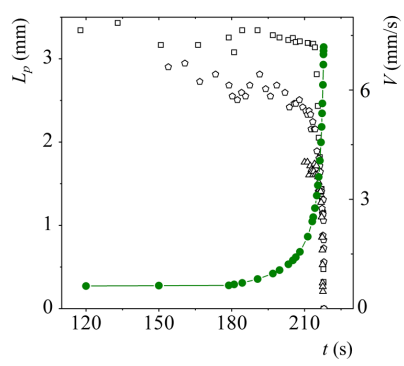

FIG. 4. Plug length $\left(L_{p}\right.$ - empty symbols) for three different plugs of carbopol $(\mathrm{C} 1)$ and mean velocity of the flow $(V$ filled symbols) as a function of time $(t)$ since the beginning of the experiment. Each plug is indicated by an empty arrow on Figure 2. The initial lengths of the plugs are $3.34 \mathrm{~mm}, 2.94$ $\mathrm{mm}$, and $1.75 \mathrm{~mm}$

For $t<200 \mathrm{~s}$ and $V<1 \mathrm{~mm} / \mathrm{s}$ the three $L_{p}$ don't exhibit any rapid variation, while for $t>200 \mathrm{~s}$, the rapid increase of $V$ induces a simultaneous catastrophic reduction of $L_{p}$ for the three plugs. This leads to the quasisimultaneous coalescence of the air bubbles. This critical dependency of the plug length with the velocity suggests that the deposition process is linked to the imposed shear rate $\left(\dot{\gamma} \sim \frac{V}{R}\right)$, and thus to the stress state of the system. For a YSF obeying to the non-linear Herschel-Bulkley law, the stress-state of the system is characterized by the Bingham number $B i^{-1}=k\left(\frac{V}{R}\right)^{n} / \tau_{y}$ which compares the effective viscous stress determined by capillary rheometry to $\tau_{y}$, the bulk yield stress. Typically when $B i^{-1}<1$ the material is mostly solid like and flows with a plug-like velocity profile, whereas for $B i^{-1}>1$ the material should be mostly liquid and the velocity profile quasi Poiseuillelike. The length of the three plugs normalized by their initial length are represented as a function of $\mathrm{Bi}^{-1}$ on Figure 5 along with other equivalent data obtained for the two model YSF.

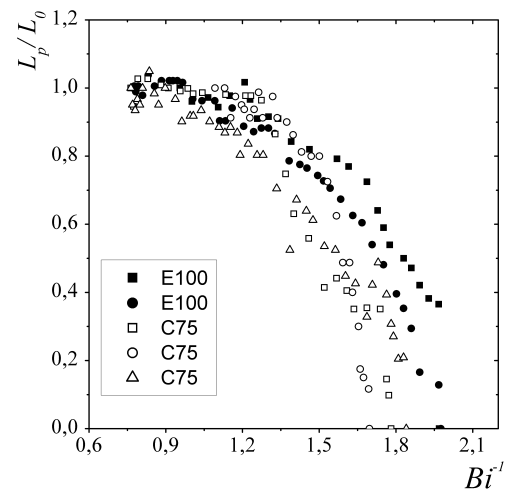

FIG. 5. The length of a plug $L_{p}$ normalized by its initial length $L_{0}$, shown as function of the inverse of the Bingham number $\left(B i^{-1}\right)$ since the beginning of the experiment for carbopol (C1 - empty symbols) and emulsion (E1 - plain symbols) plugs in a STL manufactured channel $(R=1 \mathrm{~mm}$ and $L=$ $10 \mathrm{~cm}$ )

We observe that the data obtained for different materials, and plugs with different initial size collapse reasonably on the same curve. Even if the variation of the size of the plugs depends on the history of the systems, two regimes are clearly identified: $(i)$ in the first regime, the plug size does not vary significantly. It corresponds to $B i^{-1} \leq 1$ where the viscous stress is small compared to the yield stress, and the velocity profile is mostly constituted of a solid-like region flowing like a plug and (ii) in the second regime $B i^{-1} \geq 1$, the plug size decreases rapidly (from $B i \simeq 0.8$ ) until the plugs rupture for $B i^{-1} \simeq 2$. For this range of $B i$, the material is totally fluidized and the velocity profile Poiseuille-like. As observed for Newtonian fluids, we attribute the catastrophic decrease of $L_{p}$ for $B i^{-1} \geq 1$ to film deposition along the channel's wall. To verify this point, we compute the deposited film thickness $(h)$ that surrounds the bubbles by means of a mass balance as achieved for a Newtonian fluid ${ }^{6}$.

Indeed, as a plug advances inside the channel it gathers what was deposited by the plug ahead of itself, and deposit an amount of material that depends on the bubble 
velocity $^{9,33}$. This leads to:

$$
\frac{1}{V(t)} \frac{\mathrm{d} L_{p}}{\mathrm{~d} t}=\left(1-\frac{h_{f}}{R}\right)^{2}-\left(1-\frac{h}{R}\right)^{2}
$$

Where $h_{f}$ (resp. $h$ ) denotes the thickness of the film in front of (resp. behind) the considered plug. The computation is iterative and requires the measure of the thickness of one film to be achieved. To minimize the error we choose to measure the maximum observable value of the film thickness $(\simeq 0.4 R)$. Moreover, to avoid finite size effects which may arise from interaction between the front and rear menisci of the plug and perturb the flow, we do not consider data with $L_{p}<2 R$. The results are displayed in Figure 6 as a function of $B i^{-1}$.

We interpret the plateau at low velocity as a limiting effect due to the surface roughness of the device. Due to the manufacturing process the real perimeter of the cross section of the devices is $8 R$ (see ESI for details $\dagger$ ), therefore a deposited thickness that is sufficiently thin to follow the roughness of the device would increase the interfacial energy by $27 \%$ compared to an annular film. Considering that the interfacial energy associated with the film surface must be minimized, and thus that the material must at least fill all the roughness until its surface as a curvature of $R^{-1}$, it leads to an equivalent limiting thickness $\frac{h}{R}=0.04$ (see ESI for details ${ }^{\dagger}$ ). This value is represented by the green dashed line on Figure 6, and reasonably corresponds to the measured plateau.

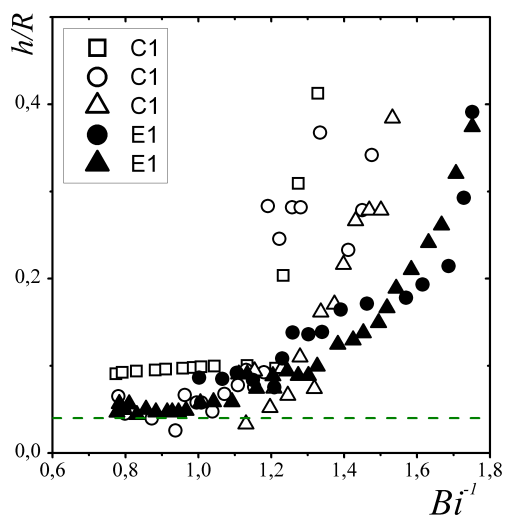

FIG. 6. Normalized film thickness $\frac{h}{R}$ as a function of $B i^{-1}$ retrieved for carbopol plugs (C1 - plain symbols) and emulsion (E1 - empty symbols) in STL devices with $R=1 \mathrm{~mm}$ and $L=$ $10 \mathrm{~cm}$. The horizontal dashed line stands the computing value of the limiting thickness imposed by the surface roughness of the devices

These results highlight that the deposition of YSF on the wall of the channel is governed by $B i^{-1}$. On the one hand, for $B i^{-1}>1$, the YSF is fluidized and the thickness of the deposited film increases with the velocity as observed recently in different geometries ${ }^{26,33,36,37,46}$. This efficient deposition process induces the rapid thinning of the plugs separating adjacent bubbles leading to their collapse. On the other hand, for $B i^{-1}>1$, the deposited thickness saturates to a value, which seems to depend on the channel roughness. In this regime, the thinning of the plug is slower, they are less prone to collapse and the start-up phase of bubble production is stable for a longer time. This suggests that the start-up phase of bubble production in channels where the roughness is extremely small, i.e. smooth channels, should be even more stable. To elucidate this point, in the following, we study the efficiency of the deposition of a YSF plug when flowing on smooth channels.

\section{Experiments using glass smooth channels.}

The stability of a single plug of either carbopol (C1) or emulsion (E2) is investigated by measuring the amount of material it lets on the wall of a smooth untreated channel (film of mean thickness $h$ ) when moving at constant velocity (steady experiment), or at imposed pressure (unsteady experiment) as sketched in Figure 2.

In the steady experiments (Figure 2a), the channel is filled with YSF and connected to a syringe mounted on a syringe pump set on withdrawal mode at fixed flow rate ( $Q_{s p}$ in range $1 \mu \mathrm{l} / \mathrm{min}-0.2 \mathrm{ml} / \mathrm{min}$ ). The fluid initially inside the capillary gradually fills the syringe, but due to the deposition of a thin film on the wall of the channel, the meniscus must move faster than the mean velocity corresponding to the imposed flow rate. We record the emptying of the capillary, and measure the velocity of the meniscus $V$. In that case, mass conservation yields to $Q_{s p}=V \pi(R-h)^{2}$, which leads to

$$
\frac{h}{R}=1-\sqrt{\frac{Q_{s p}}{\pi R^{2} V}}
$$

In the unsteady experiments (Figure 2b), a YSF plug of length $L_{p}$ is pushed inside a glass channel with a smooth wall at imposed pressure $\Delta P$. As it moves the plug deposes a thin film of YSF on the wall of the channel. This leads to a decrease in the plug length and, consequently, the plug accelerates. As performed for Newtonian fluids on rough surfaces ${ }^{2,33}$, we measure both the length of the plug $\left(L_{p}\right)$ and the velocity of its rear meniscus $(V)$ to compute the mean film thickness deposited on the wall of the channel.

Values of the film thickness $h$, obtained in the steady or unsteady experiments are shown on Figure 7 in a smooth glass capillary with $R=513 \mu \mathrm{m}$ along with data obtained in the same geometry but for no-slip boundary conditions (using treated capillaries) ${ }^{33}$. The data obtained in the two different configurations (steady and unsteady experiments) collapse on the same figure, thus validating the unsteady measurement protocol. For $Q_{s p}<0.1 \mathrm{~mL} / \mathrm{min}$ and $\Delta P \leq 5 \mathrm{mbar}$, we do not measure any significant decrease in plugs size during their motion inside the capillary $(10 \mathrm{~cm})$ : there is either no film deposited, or it is less than the resolution of our set-up (typically $1 \mu \mathrm{m}$ ). 


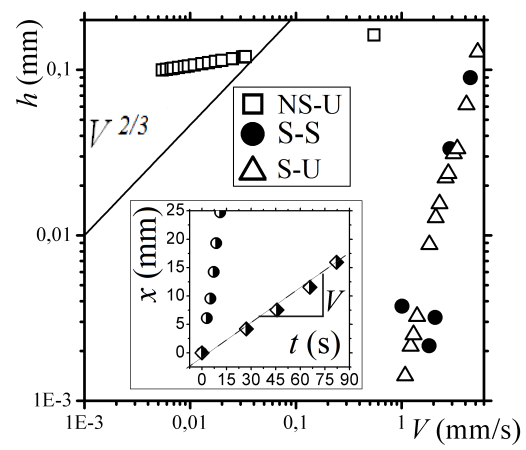

FIG. 7. Film thickness $(h)$ as a function of the velocity for: $(i)$ steady experiments with a carbopol gel (C1) in a glass channel with radius $R=513 \mu \mathrm{m}$ (filled circles). The inset shows the steady motion of the meniscus as a function of time for $Q=$ $0.1 \mathrm{ml} / \mathrm{min}$ (circles) and $Q=0.01 \mathrm{ml} / \mathrm{min}$ (diamonds); (ii) unsteady experiments a carbopol gel $(\mathrm{C} 1)$ in a glass channel $(R=513 \mu \mathrm{m})$ for no-slip boundary conditions (square) and with wall slip (triangles). The plain line stands for the typical variation for Newtonian fluids. In the legend "NS-U" stands for No slip-Unsteady, "S-U" for Slip-Unsteady, "S-S" for SlipSteady

\section{Discussion.}

On smooth surfaces, the variation of $h$ is far sharper than on rough surfaces. It typically increases with an exponent between 2 and 3 whereas it is close to 0.3 for no-slip boundary conditions, and to 0.66 for Newtonian fluids. We attribute this effect to wall slip. Indeed, wallslip is a classical feature of YSF exhibiting a microstructure. During the flow, there is a depletion of the microstructure close to the wall due to the finite size of the microelement. This region, only filled with the YSF solvent of low viscosity usually experiences most of the applied stress, thus resulting in an apparent velocity of the material at the wall ${ }^{4,7}$.

To account for the rheology of the different YSF and channels we use, we plot $\frac{h}{R}$ as a function of the inverse of the Bingham number $\left(B i^{-1}\right)$, for all the experiments performed with slip boundary conditions on Figure 8. Three regimes can be identified: $(i)$ for $B i^{-1}<1$ we are not able to measure the deposited thickness which suggests that it is either typically less than $1 \mu \mathrm{m}$ or that no deposition occurs due to wall-slip. We can note that, if a thin layer of the YSF continuous phase is deposited, this regime would last as long as the elements of the microstructure can deform and concentrate. Eventually, it would lead to the contact between the microstructure of the fluid and the wall, therefore possibly triggering (at least partial) no-slip boundary conditions and the deposition process. This may also induce stick-slip motions ${ }^{16}$. However, we did not observe such sudden deposition when the plug starts in full slip nor stick-slip motion on distances of $10 \mathrm{~cm}$; $($ ii $)$ for $B i^{-1} \sim 1$ there is sharp increase in the deposited thickness, that we interpret as a fast fluidization process due to the transition from the slip regime to the no-slip regime. Indeed, when the flow accelerates the slip layer should be destructured and the no-slip boundary conditions must be recovered; (iii) for $B i^{-1}>1$ the film thickness seems to saturate toward an almost constant value. This value roughly corresponds to what is predicted for YSF with no-slip boundary conditions ${ }^{33}$, which is expected if the slip layer is destructured.

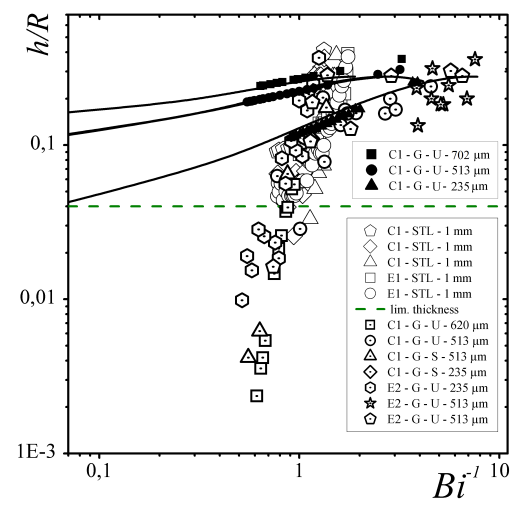

FIG. 8. Normalized film thickness $\left(\frac{h}{R}\right)$ as a function of the Bingham number for plug trains in STL channel (rough empty symbols) and glass capillaries ( $\mathrm{G}$ - dotted symbols) for different channel radii, materials, and steady $(\mathrm{S})$ or unsteady (U) experiments in channel. The legend of the experiments is detailed in the inset. Filled symbols and plain lines correspond to deposition law for no-slip boundary conditions for with channels with (from top to bottom) $R=702, \mu \mathrm{m}, 513$ $\mu \mathrm{m}, 235 \mu \mathrm{m}^{33}$

\section{Comparison with multi-plugs experiments: effect of surface roughness.}

In Figure 8, we also compare the measurements of the film thickness obtained for a single plug on a smooth surface (glass) to the results obtain for a train of plugs in STL channels (circumferantial roughness of typical size $50 \mu \mathrm{m})$.

First, we note that in the case of multi-plug experiments we do not have access to the saturation regime observed for $B i^{-1}>1$ since plugs decrease rapidly in size and rupture before $B i^{-1}>1$. Then, we observe that the measurements obtained in both set-ups exhibit similar power-law behaviour for $B i^{-1} \sim 1$. This suggests that the deposition mechanism and therefore the fluidization process of our materials is similar whatever the surface. We also underline that for glass channels, we obtain measurement of the film thickness for values that are below the geometrical limit associated with the roughness of STL devices. This is expected, since glass capillaries possess no detectable roughness and very thin film thickness can be deposited without increasing strongly the associated interfacial energy. 


\section{CONCLUSIONS}

To conclude, we have identified and studied the two main mechanisms responsible for the destabilization of bubble production in T-junction and flow focusing devices when the continuous phase is either a Newtonian or a yield-stress fluid: (i) hydrodynamic feedback due the large viscosity contrast between the continuous phase and the gas which is driven at imposed gas pressure; $(i i)$ the fluid deposition on the wall of the channel. Despite the similar mechanisms, the details are very different between Newtonian fluids and YSF. In partiular, the deposition of YSF on the wall of the channel depends on the stress state of the system (quantified by the Bingham number $B i^{-1}$ ). Three regimes of YSF deposition are thus identified: $(i)$ for $B i^{-1}<1$ the fluid slips, and the deposited layer is either very thin $(h \leq 1 \mu \mathrm{m})$ or imposed by the wall equivalent roughness: the deposition dynamics induced by the capillary pressure gradient under no-slip boundary conditions is screened. In this regime, the length of the YSF plugs does not collapse, and the flow is relatively stable; (ii) for $B i^{-1} \sim 1$ the film thickness increases rapidly. In this case the deposited film becomes thick, thus leading to the destabilization of the YSF plugs; (iii) for $B i^{-1}>1$, consistent bubble production cannot be obtained due to the quick destabilization of the bubble train. Yet, in experiments using a single plug, we show that the deposited film thickness saturates to a value that is close to that predicted by scaling laws for no-slip boundary conditions, which suggests the disappearence of the slip layer as shear rate increases (also observed in classical rheometers ${ }^{40}$ ).

These results therefore highlight the importance of the boundary conditions (in particular, channel surfaces) on the stability of YSF two-phase flows, and their interplay with surface tension (through the deposition process) and hydrodynamic effects on the stability of bubble production in microfluidic geometries. In particular, our work suggests that using slippery surfaces will potentially open new routes to controlled production of complex aerated material.

\section{ACKNOWLEDGEMENTS}

We thank L. Ducloué, J. Goyon, B. Haffner, and M. Maillard for experimental advises and discussions. We acknowledge P. Coussot and O. Pitois for fruitful discussions. This work has benefited from two French government grants managed by ANR within the frames of the national program Investments for the Future (ANR11-LABX-022-01) and of the young researcher program (ANR-11-JS09-012-WOLF).

\footnotetext{
${ }^{1}$ In supplemental material, we first provide the derivation of the area associated with the roughness of stereolithography devices as illustrated in figure S1. In figure S2, we show an enlarged picture of the channel and derive an equivalent thickness. Using the formula derived in this SM leads to a good estimate of the
}

minimum deposited quantity of yield-stress fluid for multi-plug experiments at large values of the Bingham number.

${ }^{2} \mathrm{P}$. Aussillous and D. Quéré. Quick deposition of a fluid on the wall of a tube. Physics of Fluids, 12(10):2367-2371, 2000.

${ }^{3}$ N. J. Balmforth, I. A. Frigaard, and G. Ovarlez. Yielding to Stress: Recent Developments in Viscoplastic Fluid Mechanics. Ann. Rev. Fluid Mech., 46:121-146, 2014.

${ }^{4} \mathrm{H}$. A. Barnes. A review of the slip (wall depletion) of polymer solutions, emulsions and particle suspensions in viscometers: its cause, character, and cure. J. NN Fluid Mech., 56:221 - 251, 1995.

${ }^{5}$ C. N. Baroud, F. Gallaire, and R. Dangla. Dynamics of microfluidic droplets. Lab Chip, 10:2032-2045, 2010.

${ }^{6}$ Michael Baudoin, Yu Song, Paul Manneville, and Charles N. Baroud. Airway reopening through catastrophic events in a hierarchical network. PNAS, 110:859, 2013.

${ }^{7}$ D. Bonn, J. Paredes, M. M. Denn, T. Berthier, L.and Divoux, and S. Manneville. Yield stress materials in soft condensed matter. arXiv 1502.05281, 2015.

${ }^{8} \mathrm{~J}$. Boujlel and P. Coussot. Measuring the surface tension of yield stress fluids. Soft Matter, 9:5898-5908, 2013.

${ }^{9}$ F. P. Bretherton. The motion of long bubbles in tubes. J. Fluid Mech., 10:166-188, March 1961.

${ }^{10}$ Isabelle Cantat and Renaud Delannay. Dissipative flows of $2 \mathrm{~d}$ foams. The European Physical Journal E, 18(1):55-67, 2005.

${ }^{11}$ G.F. Christopher and S.L. Anna. Microfluidic methods for generating continuous droplet streams. J. Phys. D: Appl. Phys., 40(19):319, 2007.

${ }^{12}$ Liang-Yin Chu, Andrew S Utada, Rhutesh K Shah, Jin-Woong Kim, and David A Weitz. Controllable monodisperse multiple emulsions. Angewandte Chemie International Edition, 46(47):8970-8974, 2007.

${ }^{13} \mathrm{P}$. Coussot. Yield stress fluid flows: A review of experimental data. J. N. N. Fluid Mech., pages 31-49, 2014.

${ }^{14}$ P. Coussot, L. Tocquer, C. Lanos, and G. Ovarlez. Macroscopic vs. local rheology of yield stress fluids. J. NN Fluid Mech., 158:85 $-90,2009$.

${ }^{15}$ Ladislav Derzsi, Marta Kasprzyk, Jan Philip Plog, and Piotr Garstecki. Flow focusing with viscoelastic liquids. Physics of Fluids (1994-present), 25(9):092001, 2013.

${ }^{16}$ T. Divoux, C. Barentin, and S. Manneville. From stress-induced fluidization processes to herschel-bulkley behaviour in simple yield stress fluids. Soft Matter, 7(18):8409-8418, 2011.

${ }^{17}$ A. Duboin, R. Middleton, F. Malloggi, F. Monti, and P. Tabeling. Cusps, spouts and microfiber synthesis with microfluidics. Soft Matter, 9:3041-3049, 2013.

${ }^{18}$ L. Ducloué, O. Pitois, J. Goyon, X. Chateau, and G. Ovarlez. Coupling of elasticity to capillarity in soft aerated materials. Soft Matter, 10:5093-5098, 2014.

${ }^{19}$ AM Gañán-Calvo, JM Montanero, L Martín-Banderas, and M Flores-Mosquera. Building functional materials for health care and pharmacy from microfluidic principles and flow focusing. Advanced drug delivery reviews, 65(11):1447-1469, 2013.

${ }^{20} \mathrm{P}$. Garstecki, M. J. Fuerstman, and G. M. Whitesides. Oscillations with uniquely long periods in a microfluidic bubble generator. Nature Physics, 1(3):168-171, 2005.

${ }^{21}$ P. Garstecki, I. Gitlin, W. DiLuzio, G. M. Whitesides, E. Kumacheva, and H. A. Stone. Formation of monodisperse bubbles in a microfluidic flow-focusing device. Appl. Phys. Lett., 85(13):2649-2651, 2004.

${ }^{22}$ P. Garstecki, H. A. Stone, and G. M. Whitesides. Mechanism for flow-rate controlled breakup in confined geometries: A route to monodisperse emulsions. Physical Review Letters, 94(16):164501, 2005.

${ }^{23}$ Piotr Garstecki and George M Whitesides. Flowing crystals: nonequilibrium structure of foam. Physical review letters, 97(2):024503, 2006.

${ }^{24}$ B. Geraud, L. Bocquet, and C. Barentin. Confined flows of a polymer microgel. Eur. Phys. J. E Soft Matter, 36, 2013. 
${ }^{25}$ J. Goyon, A. Colin, G. Ovarlez, A. Ajdari, and L. Bocquet. Spatial cooperativity in soft glassy flows. Nature, 454:84-87, 2008.

${ }^{26} \mathrm{C}$. Gutfinger and J. A. Tallmadge. Films of non-newtonian fluids adhering to flat plates. AIChE Journal, 11(3):403-413, 1965.

${ }^{27}$ M. Hashimoto, S. S. Shevkoplyas, B. Zasoska, T. Szymborski, P. Garstecki, and G. M. Whitesides. Formation of bubbles and droplets in parallel, coupled flow-focusing geometries. Small, 4(10):1795-1805, 2008.

${ }^{28}$ Michinao Hashimoto, Piotr Garstecki, and George M Whitesides. Synthesis of composite emulsions and complex foams with the use of microfluidic flow-focusing devices. Small, 3(10):1792-1802, 2007.

${ }^{29}$ Joung Sook Hong and Justin Cooper-White. Drop formation of carbopol dispersions displaying yield stress, shear thinning and elastic properties in a flow-focusing microfluidic channel. KoreaAustralia Rheo. J., 21:269-280, 2009.

${ }^{30}$ Axel Huerre, Vincent Miralles, and Marie-Caroline Jullien. Bubbles and foams in microfluidics. Soft Matter, 10:6888-6902, 2014.

${ }^{31}$ L. Jorgensen, M. Le Merrer, H. Delanoe-Ayari, and C. Barentin. Yield stress and elasticity influence on surface tension measurements. Soft Matter, 11:5111-5121, 2015.

${ }^{32}$ B. Laborie, F. Rouyer, D. E. Angelescu, and E. Lorenceau. Bubble formation in yield stress fluids using flow-focusing and $t$ junction devices. Phys. Rev. Lett., 114:204501, 2015.

${ }^{33}$ B. Laborie, F. Rouyer, D. E. Angelescu, and E. Lorenceau. Yieldstress fluid deposition in circular channels. submitted to J. Fluid Mech., 2016.

${ }^{34} \mathrm{C} . \mathrm{W}$. Macosko. Rheology: principles, measurements, and applications. New York: Wiley-VCH, 1994.

${ }^{35}$ F. Mahaut, X. Chateau, P. Coussot, and G. Ovarlez. Yield stress and elastic modulus of suspensions of noncolloidal particles in yield stress fluids. J. Rheol., 52, 2008.

${ }^{36}$ M. Maillard, J. Boujlel, and P. Coussot. Solid-solid transition in landau-levich flow with soft-jammed systems. Phys. Rev. Lett., 112:068304, 2014.

${ }^{37}$ M. Maillard, J. Boujlel, and P. Coussot. Flow characteristics around a plate withdrawn from a bath of yield stress fluid. J. N. N. Fluid Mech., 220:33-43, 2015.

${ }^{38}$ S. Manneville, A. Colin, G. Waton, and F. Schosseler. Wall slip, shear banding, and instability in the flow of a triblock copolymer micellar solution. Phys. Rev. E, 75:061502, 2007.

${ }^{39}$ T. G. Mason, J. Bibette, and D.A. Weitz. Yielding and flow of monodisperse emulsions. J. Colloid Interface Sci., 179:439-448, 1996.

${ }^{40}$ S. P. Meeker, R. T. Bonnecaze, and M. Cloitre. Slip and flow in pastes of soft particles: Direct observation and rheology. Journal of Rheology, 48(6):1295-1320, 2004.

${ }^{41}$ R. Mezzenga, P. Schurtenberger, A. Burbidge, and M. Michel. Understanding foods as soft materials. Nature materials, 4(10):729-740, 2005.

${ }^{42}$ P. Moller, A. Fall, V. Chikkadi, D. Derks, and D. Bonn. An attempt to categorize yield stress fluid behaviour. Phil. Trans. R. Soc. A, 367:5139-5155, 2009.

${ }^{43}$ Q.D. Nguyen and D.V. Boger. Measuring the flow properties of Yield Stress Fluids. Ann. Rev. Fluid Mech., 24:47-88, 1992.

${ }^{44}$ Zhihong Nie, MinsSeok Seo, Shengqing Xu, Patrick C. Lewis, Michelle Mok, Eugenia Kumacheva, George M. Whitesides, Piotr Garstecki, and Howard A. Stone. Emulsification in a microfluidic flow-focusing device: effect of the viscosities of the liquids. Microfluid. Nanofluidics, 5:585-594, 2008.

${ }^{45} \mathrm{~S}$ Okushima, $\mathrm{T}$ Nisisako, $\mathrm{T}$ Torii, and T Higuchi. Controlled production of monodisperse double emulsions by two-step droplet breakup in microfluidic devices. Langmuir, 20:9905-9908, 2004.

${ }^{46}$ C.-S. Park, S.-Y. Baek, K.-J. Lee, and S. W. Kim. Twophase flow in a gas-injected capillary tube. Adv. Polym. Tech., 22(4):320-328, 2003.

${ }^{47}$ J.M. Piau. Carbopol gels: Elastoviscoplastic and slippery glasses made of individual swollen sponges: Meso- and macroscopic properties, constitutive equations and scaling laws. J. NN Fluid Mech., 144:1 - 29, 2007.

${ }^{48}$ J.-P. Raven, P. Marmottant, and F. Graner. Dry microfoams: formation and flow in a confined channel. Eur. Phys. J. B, $51: 137-143,2006$

${ }^{49}$ J. R. Seth, C. Locatelli-Champagne, F. Monti, R. T. Bonnecaze, and M. Cloitre. How do soft particle glasses yield and flow near solid surfaces? Soft Matter, 8(1):140-148, 2012.

${ }^{50} \mathrm{M}$. T. Sullivan and H. A. Stone. The role of feedback in microfluidic flow-focusing devices. Phil. Trans. R. Soc. A, 1873:2131, 2008.

${ }^{51} \mathrm{G}$. A. van Aken. Aeration of emulsions by whipping. Colloids Surf, A, 190(3):333 - 354, 2001.

${ }^{52}$ Wim Van Hoeve, Benjamin Dollet, Michel Versluis, and Detlef Lohse. Microbubble formation and pinch-off scaling exponent in flow-focusing devices. Physics of Fluids (1994-present), 23(9):092001, 2011.

${ }^{53}$ T. Ward, M. Faivre, M. Abkarian, and H. A. Stone. Microfluidic flow focusing: Drop size and scaling in pressure versus flow-ratedriven pumping. Electrophoresis, 26:3716-3724, 2005.

${ }^{54}$ Ozgur E. Yildirim and Osman A. Basaran. Dynamics of formation and dripping of drops of deformation-rate-thinning and -thickening liquids from capillary tubes. J. NN Fluid Mech., 136(1):17-37, 2006. 